\title{
Cavity-Mediated Electron-Photon Superconductivity
}

\author{
Frank Schlawin, ${ }^{1, *}$ Andrea Cavalleri, ${ }^{1,2}$ and Dieter Jaksch ${ }^{1}$ \\ ${ }^{1}$ Clarendon Laboratory, University of Oxford, Parks Road, Oxford OX1 3PU, United Kingdom \\ ${ }^{2}$ Max Planck Institute for the Structure and Dynamics of Matter, Luruper Chaussee 149, 22761 Hamburg, Germany
}

(Received 21 May 2018; revised manuscript received 6 February 2019; published 4 April 2019)

\begin{abstract}
We investigate electron paring in a two-dimensional electron system mediated by vacuum fluctuations inside a nanoplasmonic terahertz cavity. We show that the structured cavity vacuum can induce long-range attractive interactions between current fluctuations which lead to pairing in generic materials with critical temperatures in the low-kelvin regime for realistic parameters. The induced state is a pair-density wave superconductor which can show a transition from a fully gapped to a partially gapped phase-akin to the pseudogap phase in high- $T_{c}$ superconductors. Our findings provide a promising tool for engineering intrinsic electron interactions in two-dimensional materials.
\end{abstract}

DOI: 10.1103/PhysRevLett.122.133602

Pairing between fermionic quasiparticles in solids through the exchange of virtual bosonic excitations is one of the most studied phenomena in the solid state because it can lead to remarkable emergent phases of matter like superconductivity. This phenomenon has been extensively investigated for the case of attractive interactions induced by phonons [1], magnetic excitations [2], and theoretically by longitudinal photons through the KohnLuttinger effect $[3,4]$. Vacuum fluctuations of the transverse electromagnetic field can also provide attractive interactions, albeit with far lower efficiency. These currentcurrent interactions in the Fermi liquid have to date been discussed in free space, where effects are restricted to very low temperatures [5-9], or in strongly correlated materials, where they could lead to the pairing of spinons [10,11]. However, interactions between current fluctuations and vacuum excitations of the electromagnetic field can occur within a cavity over mode volumes far below the free-space diffraction limit $\lambda^{3}$ (e.g., $V \sim 10^{-5} \lambda^{3}$ in Ref. [12]). These conditions are routinely created in nanoplasmonic cavities in the terahertz regime [12-19], where the ultrastrongcoupling regime between light and matter can be reached even in bad cavities. This compression of the cavity field increases the vacuum field strength $(\sim 1 / \sqrt{V})$, and thereby enhances the induced interaction $(\sim 1 / V)$ to an experimentally accessible regime.

Furthermore, the cavity structures the electromagnetic vacuum by inducing a photonic band gap $\hbar \omega_{0}$ and creating an effective photon mass $\hbar \omega_{0} / c^{2}$ (where $c$ is the speed of light in the cavity filling material) that reduces its group velocity. The extremely low-temperature scale of the effect in free space, which is attributed to the smallness of the Fermi velocity with respect to the speed of light [8], should thus be enhanced further. In contrast to polariton-mediated superconductivity [20-22], where the glue for electron pairing is provided by the exchange of hybridized excitonphoton or phonon-polariton [23] quasiparticles in a cavity, the direct interaction with a cavity does not require the pumping of the cavity, and is also not affected by excitonic interactions.

In this Letter, we show that the coupling of a terahertz cavity field to intraband transitions of a two-dimensional electron gas gives rise to a superconducting instability with a critical temperature that can reach the low-kelvin regime using realistic cavity and material parameters.

We consider a two-dimensional electron gas placed into a cavity made of two mirrors [see Fig. 1(a)]. The electronic Hamiltonian is given by $H_{0}=\sum_{\vec{k}, \sigma} \epsilon_{\vec{k}} c_{\vec{k} \sigma}^{\dagger} c_{\vec{k} \sigma}$, where $c_{\vec{k} \sigma}$ destroys an electron with quasimomentum $\vec{k}$ and polarization $\sigma$, and the electron dispersion $\epsilon_{\vec{k}}$ is determined by the lattice geometry. The cavity is described by the Hamiltonian $H_{f}=\sum_{\vec{q}, s} \hbar \omega_{q} a_{\vec{q}, s}^{\dagger} a_{\vec{q}, s}$, where $a_{\vec{q}, s}$ describes the annihilation operator of a photon with in-plane momentum $\vec{q}$ and polarization $s$, and with dispersion $\omega_{q}=$ $\omega_{0}\left[1+\left(c|\vec{q}| / \omega_{0}\right)^{2}\right]^{1 / 2}[24-26]$. To leading order, the interaction between cavity and electron system is given by the paramagnetic coupling of the form $[27,28]$

$$
H_{\mathrm{int}}=\sum_{\vec{k}, \sigma \vec{q}, s} \frac{g_{\vec{k}, s}^{(\vec{q})}}{\sqrt{N}}\left(a_{\vec{q}, s}+a_{-\vec{q}, s}^{\dagger}\right) c_{\vec{k}+\vec{q}, \sigma}^{\dagger} c_{\vec{k}, \sigma},
$$

where $g_{\vec{k}, s}^{(\vec{q})}$ is a coupling parameter and $N$ indicates the number of sites. We model the cavity mode compression below the diffraction limit by a compression factor $A$, such that $g \propto 1 / \sqrt{A}$ [see Supplemental Material (SM) [29]]. Note that we have not invoked the commonly used dipole approximation, nor the rotating wave approximation which 


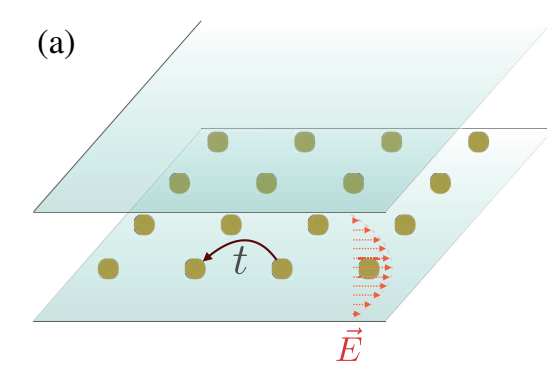

(c)

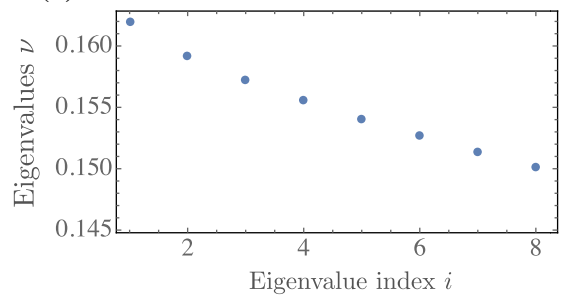

(b1)

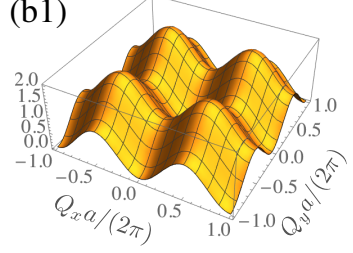

(b2)

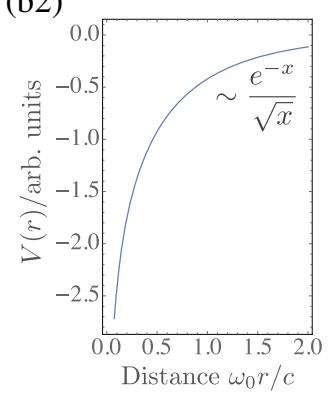

(d)
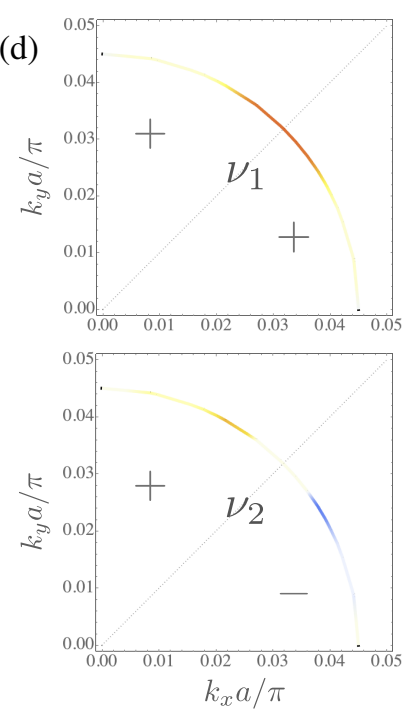

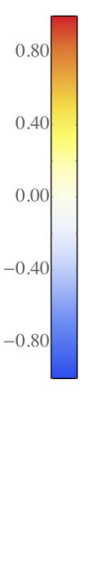

FIG. 1. (a) A 2D electron gas depicted by yellow sites interacts with a cavity, whose electric field distribution is indicated in red. The electronic hopping amplitude $t$ is also sketched. (b) The exchange of virtual photons between electric intraband transitions creates an effective electron interaction. This is decomposed into two contributions as described in the main text: panel (b1) displays the effect of the lattice geometry on the cavity-mediated interaction across the first Brillouin zone as a function of the nesting vector $\vec{Q}$ and panel (b2) shows the interaction Eq. (4) in arbitrary units (arb. units). (c) Leading eigenvalues $\nu_{i}$ according to Eq. (5). (d) The eigenfunctions corresponding to eigenvalues $\nu_{1}$ and $\nu_{2}$ pertaining to a nesting vector $\vec{Q}=k_{0}(1,1)$ are shown along the positive quadrant of the Fermi surface, and positive and negative sectors of the wave functions are indicated. The color code is given on the right-hand side. $\nu_{1}$ corresponds to a singlet and $\nu_{2}$ to triplet symmetry. We used parameters appropriate for a $2 \mathrm{D}$ electron gas in GaAs: relative permittivity $\epsilon=13$, electron band mass $m^{*}=0.069 m_{e}$, chemical potential $\mu=-3.98 t$, lattice constant $a=5.6 \AA$, and a cavity frequency $\omega_{0}=2 \pi \times 5 \mathrm{THz}$.

can fail in the strong-coupling regime [30]. While our calculations are based on a specific model for the cavity geometry shown in Fig. 1(a) (details are given in SM [29]), we expect the main results of our Letter to be independent of the specific details of the cavity mode.

Integrating out the cavity degrees of freedom, which are in their ground state, and using that $\hbar \omega_{q} \gg\left|\epsilon_{\vec{k}}-\epsilon_{\vec{k}}\right|$, the interaction Hamiltonian Eq. (1) yields the cavity-mediated electron interaction

$V_{\text {eff }}=-\frac{1}{N} \sum_{\vec{k}, \sigma, \vec{k}^{\prime}, \sigma^{\prime}} \sum_{\vec{q}, s} \frac{g_{\vec{k}, s}^{(\vec{q})} g_{\vec{k}^{\prime}, s}^{(-\vec{q})}}{\hbar \omega_{q}} c_{\vec{k}+\vec{q}, \sigma}^{\dagger} c_{\vec{k}, \sigma} c_{\vec{k}^{\prime}-\vec{q}, \sigma^{\prime}}^{\dagger} c_{\vec{k}^{\prime}, \sigma^{\prime}}$

Equation (2) describes the interaction between current fluctuations mediated by the structured electromagnetic vacuum. Because of the symmetry of the electromagnetic field, this interaction is attractive for electrons propagating in the same direction. This "Amperean" pairing instability can give rise to superconductivity with the Cooper pairs residing on the same side of the Fermi surface (FS), and having a large center-of-mass momentum $\sim 2 k_{f}$, where $k_{f}$ is the Fermi wave vector $[10,11]$. The resulting pair-density superconductor has been discussed in the pseudogap phase of cuprate superconductors [31] and in topological insulators [32], yet it has never been identified unambiguously to the best of our knowledge.
Amperean pairing can lead to the condensation of electron pairs in the vicinity of a nesting vector $\vec{Q}$ on the Fermi surface [10]. Relabeling the indices, $\vec{k}=\vec{Q}+\vec{p}^{\prime}, \vec{k}^{\prime}=\vec{Q}-\vec{p}^{\prime}$, and $\vec{q}=\vec{p}-\vec{p}^{\prime}$, the cavitymediated electron interaction Eq. (2) reads $V_{\text {eff }}=$ $(1 / 2 N) \sum_{Q, \vec{p}, p^{\prime}} \quad \sum_{\sigma, \sigma^{\prime}} K_{\vec{p}, p^{\prime}}^{(Q)} \epsilon_{Q \vec{\gamma} p, \sigma}^{\dagger} \epsilon_{Q \vec{Q} p, \sigma^{\prime}}^{\dagger} e_{Q \overrightarrow{-} p^{\prime}, \sigma^{\prime}} e_{Q \overrightarrow{+} p^{\prime}, \sigma^{\prime}}$, with $V_{\vec{p} \vec{p}^{\prime}}^{(\vec{Q})}=-2 \sum_{s} g_{\vec{Q}+\vec{p}^{\prime}, s}^{\left(\vec{p}-\vec{p}^{\prime}\right)} g_{\vec{Q}-\vec{p}^{\prime}, s}^{\left(\overrightarrow{p^{\prime}}-\vec{p}\right)} / \hbar \omega_{\left|\vec{p}-\vec{p}^{\prime}\right|}$. In the following, we will investigate this attractive interaction on a simple rectangular lattice with nearest-neighbor hopping $t$, for which the electron dispersion reads $\epsilon_{\vec{k}}=$ $-2 t\left[\cos \left(k_{x} a\right)+\cos \left(k_{y} a\right)\right]-\mu$, where $a$ denotes the lattice constant, and where we also added the chemical potential $\mu$. We find (see SM [29])

$V_{\vec{p}, \vec{p}^{\prime}}^{(\vec{Q})}=-\frac{V_{0}}{2} \frac{\sum_{i=x, y}\left\{\cos \left[\left(p_{i}+p_{i}^{\prime}\right) a\right]-\cos \left[2 Q_{i} a\right]\right\}}{\left(\omega_{0} a / c\right)^{2}+\left[\left(\vec{p}-\vec{p}^{\prime}\right) a\right]^{2}}$,

where we defined the overall interaction strength $V_{0}=$ $2 g_{0}^{2}\left(a \omega_{0} / c\right)^{2} / \hbar \omega_{0}$, and $g_{0}$ denotes the coupling strength which contains the cavity compression. The interaction Eq. (3) is attractive, as long as $\cos \left[\left(p_{i}+p_{i}^{\prime}\right) a\right]>$ $\cos \left[2 Q_{i} a\right]$. The current operator conserves the electron spin, and as such, it can facilitate electron pairing in both the singlet and the triplet channel. 
The potential $V_{\vec{p}, \vec{p}^{\prime}}^{(\vec{Q})}$ peaks sharply around $\vec{p}=\vec{p}^{\prime}$, such that it can affect only a very narrow band around the Fermi energy. The width of this peak is determined by the cavity properties encoded in the denominator, which only depends on $\left|\vec{p}-\vec{p}^{\prime}\right|$. In the corresponding coordinate space $r$, the potential is proportional to the zeroth-order Bessel function of the second kind,

$$
V(r) \sim-K_{0}\left(\frac{\omega_{0} r}{c}\right)
$$

shown in Fig. 1(b2). It shows a logarithmic divergence at the origin, and tails decaying as $\sim \exp \left(-\omega_{0} r / c\right) /\left(\omega_{0} r / c\right)^{1 / 2}$. The exponential decay is a consequence of the off-resonant coupling. At larger distances, the virtual photons take on a real character [33], and the interaction is suppressed by energy conservation. However, the length scale of this decay $c / \omega_{0}$ is $\sim \mu \mathrm{m}$ for terahertz cavities, and thus up to 4 orders of magnitude larger than typical lattice constants $a \sim 10 \AA$. For all practical purposes, the cavity-mediated interaction can therefore be considered a long-range interaction.

The numerator of $V_{\vec{p}, \vec{p}^{\prime}}^{(\vec{Q})}$ depends solely on $\left(\vec{p}+\vec{p}^{\prime}\right)$ and the nesting vector $\vec{Q}$. For small $\vec{p}$ and $\vec{p}^{\prime}$, it reduces to $\sim \sin ^{2}\left(Q_{x} a\right)+\sin ^{2}\left(Q_{y} a\right)$, which is plotted in Fig. 1(b1). Clearly, the interaction is strongest along the (anti)diagonals $Q_{y}= \pm Q_{x}$. So in the following, we will investigate pairing in the vicinity of the points $\vec{Q}=k_{0}( \pm 1, \pm 1)$, where $k_{0}$ is chosen such that $\vec{Q}$ lies on the Fermi surface.

We decouple the electron interactions with the mean fields $\Delta_{\sigma \sigma^{\prime}}^{(\vec{Q})}(\vec{p})=N^{-1} \sum_{\vec{p}^{\prime}} V_{\vec{p}, \vec{p}^{\prime}}^{(\vec{Q})}\left\langle c_{\vec{Q}-\vec{p}^{\prime}, \sigma^{\prime}} c_{\vec{Q}+\vec{p}^{\prime}, \sigma}\right\rangle$. Since $V_{\vec{p}, \vec{p}^{\prime}}^{(\vec{Q})}$ is attractive only in the vicinity of $\vec{Q}$, we can evaluate pairing around the different nesting vectors separately, and each of these turns into an identical calculation. To identify the dominating gap symmetry, we follow the approach in Refs. [34,35] adopted for the pair-density wave state (see SM [29]): We linearize the gap equation near the critical temperature $T_{c}$, and determine the largest eigenvalues $\nu$ of the equation

$$
-\frac{1}{2} \int_{\vec{Q}+\vec{p}^{\prime} \in F S} \frac{d \vec{p}^{\prime}}{(2 \pi)^{2}} \frac{1}{\left|\vec{v}\left(\vec{p}^{\prime}\right)\right|} V_{\vec{p}, \vec{p}^{\prime}}^{(\overrightarrow{\vec{Q})}} \Delta^{(\vec{Q})}\left(\vec{p}^{\prime}\right)=\nu \Delta^{(\vec{Q})}(\vec{p}),
$$

where the line integral runs over vectors $\vec{p}$, such that $\vec{Q}+\vec{p}$ is on the Fermi surface, and $\vec{v}(\vec{p})=$ $\left(\nabla_{\vec{Q}+\vec{p}} \epsilon_{\vec{Q}+\vec{p}}+\nabla_{\vec{Q}-\vec{p}} \epsilon_{\vec{Q}-\vec{p}}\right) / 2$. We discretize the quadrant of the FS containing $\vec{Q}$, and solve the matrix equation iteratively with the Arnoldi algorithm implementation in Mathematica. The critical temperature is then given by $k_{B} T_{c}=1.13 \hbar \omega_{c} e^{-1 / \nu}$ [36], where $\omega_{c}$ denotes the cutoff frequency, which we set equal to the basic cavity frequency $\omega_{0}$. In the following, we use values appropriate for a twodimensional electron gas in GaAs heterostructures, keeping in mind that the critical temperature will also be affected by details of the cavity field (see SM [29]). The GaAs system is well described by an effective mass description of the single electrons [37], and phonon scattering is negligible below $1 \mathrm{~K}$ [38]. Using a cavity compression $A=2 \times 10^{-5}$ (i.e., $\left.V \sim 2 \times 10^{-5}(\lambda /(2 \sqrt{\varepsilon}))^{3}\right)$ in Fig. 1(c), we obtain dimensionless coupling strengths $\nu \sim 0.16$. This translates into possible critical temperatures reaching into the lowkelvin regime (see also SM for discussion [29]). We have thus established that cavity-mediated electron interactions in electron gases could readily be detected with existing terahertz cavity technology. Since cavity mode volumes as small as $10^{-7}(\lambda / \sqrt{\varepsilon})^{3}$ have been reported recently [19] (although at subterahertz frequencies), higher critical temperatures are conceivable. A strong-coupling extension of our theory will be required in this case, since the eigenvalues of Eq. (5) could then reach values close to unity. In the remainder of this Letter, we shall be concerned with the unusual properties and consequences of the fact that the electron pairing here is controlled externally by properties of the cavity.

The dependence of the pairing strength on the electron density $n_{e}$ is investigated in Fig. 2(a), where we plot the leading eigenvalue $\nu_{1}$ vs $n_{e}$ for a fixed cavity compression factor $A=10^{-5}$ over a large range of densities. An increase of the electron density by a factor 10 translates into a similar change of $\nu_{1}$. Hence, the cavity compression factor can always outweigh a change of the electron density, and pairing should be observable even in a low-filling regime with $n_{e} \lesssim 10^{11} \mathrm{~cm}^{-2}$ - given a sufficiently strong cavity compression to push the leading eigenvalue to $\sim 0.1$. In the following, we fix the largest eigenvalue at a value corresponding to a zero-temperature mean field value $\Delta_{0}$, and investigate the gap structure at different electron densities. The Amperean pairing around a nesting vector $\vec{Q}$ gives rise to a spatial modulation of the superconducting order parameter in real space, $\Delta(\vec{r}) \sim \cos (\vec{Q} \cdot \vec{r})$ (assuming equal pairing amplitude on opposite sides of the Fermi surface, i.e., for $\vec{Q}$ and $-\vec{Q}$ ). The electron density determines $\vec{Q}$, and hence should strongly influence the emerging gap structure.

In Fig. 2(b), the quasiparticle dispersion curves $E(\vec{k})$ (see SM [29]) of the two highest-energy states are plotted vs $k_{x}$ along two cuts, $k_{y}=0$ and $k_{y}=k_{f}$, respectively. Evidently, the dispersion is not symmetric with respect to the Fermi energy, as the particle-hole symmetry is broken in the pair-density wave state, and the hole dispersion is split into two maxima at $\pm Q_{x} / 2$. Still, at this low electron density, the quasiparticle spectrum is gapped along the whole Fermi surface. At larger electron densities, as shown in In Fig. 2(c), the nesting vector $\vec{Q}$ increases with the Fermi energy, and so does the split in the hole dispersion. As a consequence, the gap is closed along parts of the Fermi surface (e.g., at $k_{y}=k_{f}$ ). This can be understood by reference to Fig. 1(b1): At higher electron densities, the coupling strength becomes more anisotropic. Hence, the 

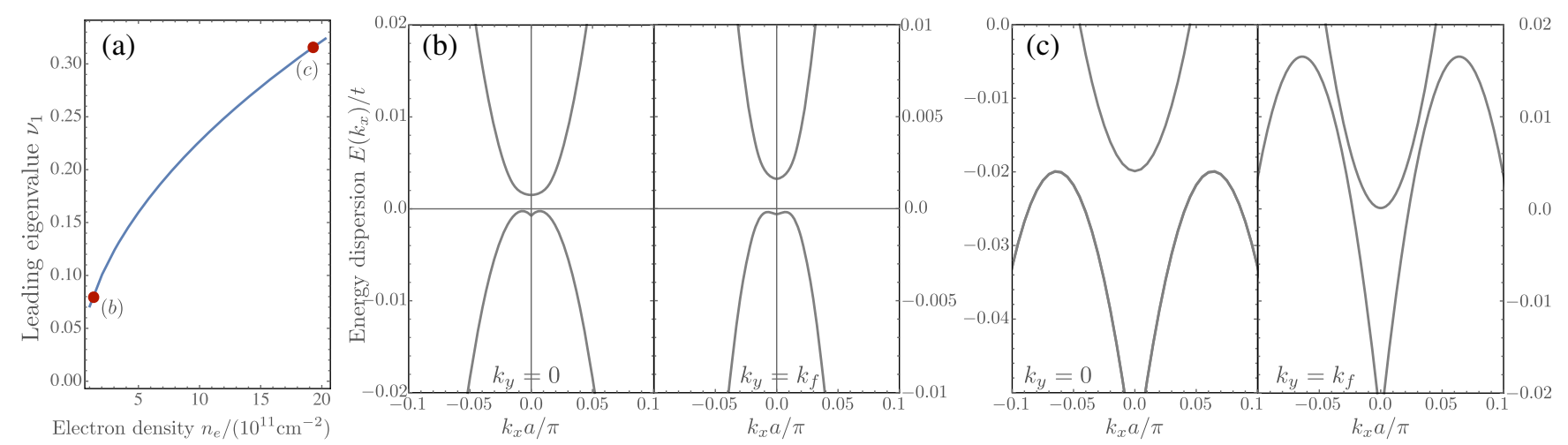

FIG. 2. (a) The leading eigenvalue of Eq. (5) is plotted as a function of the electron density $n_{e}$, corresponding to chemical potentials $\mu \in[-3.999,-3.98] t$, and a cavity compression factor $A=10^{-5}$. (b) Electron dispersion $E\left(k_{x}\right)$ along $k_{x}$, with $k_{y}=0$ (left) and $k_{y}=k_{f}$ (right) for low electron density $n_{e} \simeq 0.3 \times 10^{11} \mathrm{~cm}^{-2}$, and $\Delta_{0}=10^{-3} t$. The axes $E(0)$ and $k_{x}=0$ are indicated for orientation. The system is fully gapped everywhere along the Fermi surface. (c) Same as (b) with larger electron density $n_{e} \simeq 2 \times 10^{12} \mathrm{~cm}^{-2}$, and $\Delta_{0}=10^{-3} t$. It is gapped only along certain directions in reciprocal space.

condensate is created in the close vicinity of the nesting vectors. Conversely, at low densities, the coupling becomes isotropic. The eigenfunction in Fig. 1(d) broadens, and the entire Fermi surface is gapped.

In cuprate superconductors, this pair-density wave state is considered a possible candidate for the pseudogap phase [31], where parts of the Fermi surface remain gapped, while others are not-giving rise to the Fermi arc in angleresolved photoemission spectroscopy measurements [39]. Cavity-mediated Amperean pairing in GaAs heterostructures shows the same phenomenology in a much cleaner system. By varying the electron density, it is possible to induce a change between a fully gapped low-density and a partially gapped high-density state.

So far, we have only considered the dominating eigenvalue obtained from Eq. (5). Yet the eigenvalue spectrum shown in Fig. 1(c) shows a succession of eigenvalues with decreasing amplitude which are separated by less than $1 \%$ of their absolute values. The eigenfunctions corresponding to the two leading eigenvalues are shown in Fig. 1(d), and correspond to a singlet order, with $\Delta_{-\vec{p}}^{(\vec{Q})}=\Delta_{\vec{p}}^{(\vec{Q})}$, and a triplet order, with $\Delta_{-\vec{p}}^{(\vec{Q})}=-\Delta_{\vec{p}}^{(\vec{Q})}$, respectively. Since the cavity-mediated pairing potential of two electrons is longrange compared to electronic length scales, a node (as in triplet pairing) in the two-electron wave function only results in a small energy penalty, thus pushing the different orders towards degeneracy. Up to now, we neglected the screened Coulomb repulsion. Yet in a real material, the interaction potential between two electrons will look rather like the sketches inset in Fig. 3(b): the long-range cavitymediated interaction dominates at large distances, but at short distances, other intrinsic interactions of the electron gas affect the pairing potential. The singlet wave function peaks at zero distance, and should be influenced strongly by local repulsive interactions. On the other hand, the triplet state vanishes at the origin, and should thus be affected less strongly. In the current situation, where different eigenvalues are separated by only a few percent, these local interactions could decide the realized state. We will examine the simplest possible local interaction in the following: a constant Hubbard- $U$ describing a local repulsive electron interaction. We replace the original interaction in Eq. (5) with $V_{\vec{p}, \vec{p}^{\prime}}^{(\vec{Q})}+U$ and investigate the leading eigenvalues of the resulting effective interaction.

Figure 3(a) shows the largest positive eigenvalue of the matrix eigenvalue equation (5) as a function of the cavity enhancement $A^{-1}$, thus modulating the interaction strength. The eigenvalue grows linearly with $A^{-1}$, and shows no discernible structure. In Fig. 3(b), we show the normalized

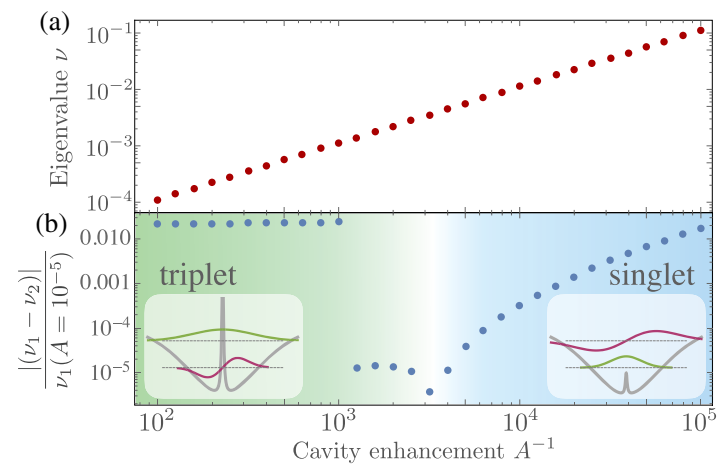

FIG. 3. (a) The leading positive eigenvalue of Eq. (5) is plotted as a function of the cavity enhancement $A^{-1}$, for $\mu=-3.99 t$. (b) Absolute value of the difference between the leading $\nu_{1}$ and subleading eigenvalue $\nu_{2}$, normalized to the leading eigenvalue at $A=10^{-5}$. At low enhancements, $\nu_{1}$ is negative (and the $s$ wave repulsive), resulting in large differences. The inset on the left sketches the effective interaction potential in this region in gray. The green wave function sketches the $s$-wave function which is strongly affected by the local repulsion, and thus suppressed energetically. Hence, the $p$ wave (red) is energetically favorable, and pairing occurs in the triplet channel. On the right, the repulsion is too weak to suppress the $s$ wave. 
difference between the two dominating eigenvalues of the matrix eigenvalue equation (5), revealing a structural change with $A^{-1}$. At very low enhancements, the difference is rather large. In this regime, the singlet state is negative (repulsive), and the system is dominated by triplet pairing. Then, at $A^{-1} \simeq 10^{3}$, the singlet state becomes attractive; the difference to the triplet state shrinks, and decreases until the cavity compression reaches $A^{-1} \simeq 2 \times 10^{3}$, where the singlet order becomes larger than the triplet component. Thus, with increasing coupling strength, the system switches from a low-temperature triplet regime to a singlet regime at strong couplings. In GaAs heterostructures, however, this transition takes place at very low temperatures, and it appears difficult to observe the triplet phase in this system. It could possibly be observed in complex oxide interfaces [37] with smaller electron mobilities and larger electron densities $\sim 10^{15} \mathrm{~cm}^{-2}$.

Our results open a new route towards the optical manipulation of superconductivity. The cavity-induced modification of the electronic ground state-similar to experiments in molecular systems [40-43], and related theoretical proposals to employ cavities to change molecular dynamics [44-51] and ground states [52,53]—could prove to be a very powerful tool to enhance or design coherent electronic states in two-dimensional materials. Furthermore, we have not considered possible external driving of the cavity. Driving the cavity into a nonlinear intensity regime would certainly influence the cavitymediated interactions, and might possibly be able to stabilize electron pairing at higher temperatures, analogous to the enhancement observed when materials are driven with strong coherent fields at terahertz frequencies [54-56].

To conclude, we have investigated electron interactions mediated by a terahertz cavity strongly coupled to a twodimensional electron system. These long-range interactions arise-very much like van der Waals or Casimir-Polder forces between macroscopic bodies [57]-from the exchange of virtual photons between spontaneous current fluctuations. Since the critical temperature scales approximately linearly with the cavity frequency, we suggest that Ref. [13], for instance, conducted their measurements above $T_{c}$, and thus did not observe the effects we have predicted here. We discuss the impact of very weak disorder in the SM [29], yet stronger disorder could further reduce $T_{c}$. The counterrotating terms in the electron-photon coupling, which the present theory relies upon, were recently reported in Ref. [30], demonstrating the feasibility of observing cavity-mediated interactions.

The authors would like to thank Giacomo Mazza for helpful discussions. The research leading to these results has received funding from the European Research Council under the European Union's Seventh Framework Programme (FP7/2007-2013) Grant Agreement No. 319286 Q-MAC. *frank.schlawin@physics.ox.ac.uk

[1] J. P. Carbotte, Rev. Mod. Phys. 62, 1027 (1990).

[2] P. A. Lee, N. Nagaosa, and X. G. Wen, Rev. Mod. Phys. 78, 17 (2006).

[3] W. Kohn and J. M. Luttinger, Phys. Rev. Lett. 15, 524 (1965).

[4] J. González, Phys. Rev. B 78, 205431 (2008).

[5] T. Holstein, R. E. Norton, and P. Pincus, Phys. Rev. B 8, 2649 (1973).

[6] M. Yu. Reizer, Phys. Rev. B 39, 1602 (1989).

[7] M. Yu. Reizer, Phys. Rev. B 40, 11571 (1989).

[8] D. V. Khveshchenko, Phys. Rev. B 47, 3446 (1993).

[9] D. V. Khveshchenko, R. Hlubina, and T. M. Rice, Phys. Rev. B 48, 10766 (1993).

[10] S.-S. Lee, P. A. Lee, and T. Senthil, Phys. Rev. Lett. 98, 067006 (2007).

[11] V. Galitski and Y. B. Kim, Phys. Rev. Lett. 99, 266403 (2007).

[12] C. Maissen, G. Scalari, F. Valmorra, M. Beck, J. Faist, S. Cibella, R. Leoni, C. Reichl, C. Charpentier, and W. Wegscheider, Phys. Rev. B 90, 205309 (2014).

[13] G. Scalari et al., Science 335, 1323 (2012).

[14] X. Gan, K. F. Mak, Y. Gao, Y. You, F. Hatami, J. Hone, T. F. Heinz, and D. Englund, Nano Lett. 12, 5626 (2012).

[15] X. Liu, T. Galfsky, Z. Sun, F. Xia, E.-c. Lin, Y.-H. Lee, S. Kéna-Cohen, and V. M. Menon, Nat. Photonics 9, 30 (2015).

[16] S. Smolka, W. Wuester, F. Haupt, S. Faelt, W. Wegscheider, and A. Imamoglu, Science 346, 332 (2014).

[17] Q. Zhang, M. Lou, X. Li, J. L. Reno, W. Pan, J. D. Watson, M. J. Manfra, and J. Kono, Nat. Phys. 12, 1005 (2016).

[18] A. Bayer, M. Pozimski, S. Schambeck, D. Schuh, R. Huber, D. Bougeard, and C. Lange, Nano Lett. 17, 6340 (2017).

[19] J. Keller, G. Scalari, S. Cibella, C. Maissen, F. Appugliese, E. Giovine, R. Leoni, M. Beck, and J. Faist, Nano Lett. 17, 7410 (2017).

[20] F. P. Laussy, A. V. Kavokin, and I. A. Shelykh, Phys. Rev. Lett. 104, 106402 (2010).

[21] O. Cotlet, S. Zeytinoglu, M. Sigrist, E. Demler, and A. Imamoglu, Phys. Rev. B 93, 054510 (2016).

[22] A. Kavokin and P. Lagoudakis, Nat. Mater. 15, 599 (2016).

[23] M. A. Sentef, M. Ruggenthaler, and A. Rubio, Sci. Adv. 4, eaau6969 (2018).

[24] K. Kakazu and Y. S. Kim, Phys. Rev. A 50, 1830 (1994).

[25] Y. Todorov, Phys. Rev. B 91, 125409 (2015).

[26] D. Hagenmüller, S. De Liberato, and C. Ciuti, Phys. Rev. B 81, 235303 (2010).

[27] M. Fox, Optical Properties of Solids (Oxford University Press, Oxford, 2001).

[28] M. Dressel and G. Grüner, Electrodynamics of Solids: Optical Properties of Electrons in Matter (Cambridge University Press, Cambridge, England, 2002).

[29] See Supplemental Material at http://link.aps.org/ supplemental/10.1103/PhysRevLett.122.133602 for details on the field quantization, the derivation of Eqs. (1)-(3), and the influence of weak disorder.

[30] X. Li et al., Nat. Photonics 12, 324 (2018).

[31] P. A. Lee, Phys. Rev. X 4, 031017 (2014). 
[32] M. Kargarian, D. K. Efimkin, and V. Galitski, Phys. Rev. Lett. 117, 076806 (2016).

[33] A. Salam, Molecular Quantum Electrodynamics (John Wiley \& Sons, Hoboken, 2010).

[34] D. J. Scalapino, E. Loh, and J. E. Hirsch, Phys. Rev. B 34, 8190 (1986).

[35] A. T. Rømer, A. Kreisel, I. Eremin, M. A. Malakhov, T. A. Maier, P. J. Hirschfeld, and B. M. Andersen, Phys. Rev. B 92, 104505 (2015).

[36] V. P. Mineev and M. Sigrist, Basic theory of Superconductivity in Metals without Inversion Center, in NonCentrosymmetric Superconductors: Introduction and Overview (Springer, Berlin, 2012), pp. 129-154.

[37] S. Stemmer and S. J. Allen, Annu. Rev. Mater. Res. 44, 151 (2014).

[38] M. J. Manfra, Annu. Rev. Condens. Matter Phys. 5, 347 (2014).

[39] A. Damascelli, Z. Hussain, and Z.-X. Shen, Rev. Mod. Phys. 75, 473 (2003).

[40] J. A. Hutchinson, T. Schwartz, C. Genet, E. Deveaux, and T. W. Ebbesen, Angew. Chem. 51, 1592 (2012).

[41] S. Wang, A. Mika, J. A. Hutchison, C. Genet, A. Jouaiti, M. W. Hosseini, and T. W. Ebbesen, Nanoscale 6, 7243 (2014).

[42] E. Orgiu et al., Nat. Mater. 14, 1123 (2015).

[43] T. W. Ebbesen, Acc. Chem. Res. 49, 2403 (2016).

[44] J. Feist and F. J. Garcia-Vidal, Phys. Rev. Lett. 114, 196402 (2015).
[45] J. Schachenmayer, C. Genes, E. Tignone, and G. Pupillo, Phys. Rev. Lett. 114, 196403 (2015).

[46] J. Galego, F. J. Garcia-Vidal, and J. Feist, Phys. Rev. X 5, 041022 (2015).

[47] M. Kowalewski, K. Bennett, and S. Mukamel, J. Chem. Phys. 144, 054309 (2016).

[48] M. Kowalewski, K. Bennett, and S. Mukamel, J. Phys. Chem. Lett. 7, 2050 (2016).

[49] J. Galego, F. J. Garcia-Vidal, and J. Feist, Nat. Commun. 7, 13841 (2016).

[50] F. Herrera and F. C. Spano, Phys. Rev. Lett. 116, 238301 (2016).

[51] J. Galego, F. J. Garcia-Vidal, and J. Feist, Phys. Rev. Lett. 119, 136001 (2017).

[52] J. Flick, M. Ruggenthaler, H. Appel, and A. Rubio, Proc. Natl. Acad. Sci. U.S.A. 114, 3026 (2017).

[53] J. Flick, C. Schäfer, M. Ruggenthaler, H. Appel, and A. Rubio, ACS Photonics 5, 992 (2018).

[54] M. Först, C. Manzoni, S. Kaiser, Y. Tomioka, Y. Tokura, R. Merlin, and A. Cavalleri, Nat. Phys. 7, 854 (2011).

[55] A. Subedi, A. Cavalleri, and A. Georges, Phys. Rev. B 89, 220301(R) (2014).

[56] M. Mitrano et al., Nature (London) 530, 461 (2016).

[57] L. M. Woods, D. A. R. Dalvit, A. Tkatchenko, P. RodriguezLopez, A. W. Rodriguez, and R. Podgornik, Rev. Mod. Phys. 88, 045003 (2016). 